\title{
Perusing the Literature via Citation Links*
}

\author{
Miranda Lee Pao \\ School of Information and Library Studies, The University of Michigan, 550 East University, \\ Ann Arbor, Michigan 48109-1092
}

Received April 16, 1992

\begin{abstract}
While MEDLINE searching is recognized as the single most effective means to identify relevant items to solve clinical and research problems, the clinician should also consider the complementary strategy to search for relevant items citing a known key paper. This study reports on the usefulness of citation searching based on the analysis of 89 searches. For each topic, the citations linked to an average of $24 \%$ additional relevant materials. At least one relevant item was added to $85 \%$ of the searches. The additional effort of scanning another printout is minimal since citation searching for $42 \%$ of the searches produced less than 7 additional items, half of which were judged to be useful. Duplicate retrievals were mostly of definite relevance. This alternate strategy appeared to be effective in interdisciplinary topics. Furthermore, the online version of the citation index is known for short turnaround time in processing, a feature important for many rapidly developing specialties. (0) 1993 Academic Press, Inc.
\end{abstract}

\section{INTRODUCTION}

Today, MEDLINE is widely available to physicians by mediated search services offered by the library, by the ever-increasing number of passwords issued directly to health professionals by the National Library of Medicine, and by various forms of local access to the MEDLINE database. The latter group includes dial-in capabilities to subsets of the file installed on local computers, and CD-ROM files accessible on network file servers, or single-user stations of CD-ROM MEDLINE. Furthermore, easy access has been facilitated by a number of end-user MEDLINE search systems, including the easy-to-use interface, GRATEFUL MED, developed by the National Library of Medicine.

In a series of papers, Haynes and his colleagues have strongly recommended MEDLINE as an effective means to identify relevant items in solving clinical problems $(I)$. Nevertheless, effective MEDLINE searching requires an investment of time and effort in learning the file content, record structure, and the ways document content are represented as well as the mechanics of the specific

* This work was supported by the NLM Grant R01-LM-04177 from the National Library of Medicine. 
search system (2-4). Their results also showed that different searchers searching on the identical topic retrieved largely different output sets. Previously overlap studies in the information retrieval literature have reported similar results $(5-8)$. Saracevic commented that "different searchers for the same question see and interpret different things in a question, represent them by different linguistic and/or logical constructs, and retrieve different things from a file (7). Furthermore, many reported that different items were retrieved by using terms taken from the abstracts, titles, and/or assigned subject headings fields of bibliographic records (9). In other words, the relationship existing between the concepts contained in a document and the terms used to represent them is not a fixed and absolute one. Indexers do not choose the same words to represent a given concept; different searchers approach a search topic differently. Consequently, subject retrieval does not appear to be as straight forward as one might expect.

In actuality, effective information seekers make use of a number of strategies. Many would never miss reading whatever is published by a few known experts in the field. Regular critical reading of a few core journals is a favorite strategy for many. Tracking down items cited in the bibliographies is another way to locate relevant items. Some swear by the reliability of current awareness or selective dissemination of information services (SDI) to keep them informed. Enterprising individuals even search recent papers which have cited a few important works or individuals. Still others might even have the luxury of browsing through the stacks or the current journal shelves in hopes of finding information nuggets serendipitously.

Among the many alternative ways to seek useful information, citation searching offers a viable route to subject retrieval. As early as 1971, Salton, a pioneer in automatic information retrieval, found in a small file that searching by terms and by citation links produced complementary sets (10). The increase in the number of relevant items added to a traditional search was as high as $10 \%$. Since then experiments from small experimental files, often using experimental procedures, have shown that citation relations can add to term searching (5). However, none of these experiments was able to compare subject retrieval by terms and by citations in operational settings, using commercially available databases with real searches. Few have attempted to assess the extent of incremental relevant retrieval by citations.

In this paper, the potential of literature searching by citation relations is examined in the context of a study that compared 89 search topics processed in health sciences libraries. Each topic was searched by the use of subject terms and by citations. This paper reports on the extent to which citation searches could add to the relevant pool of items retrieved from MEDLINE on the same search topic. Benefits in the use of a second database are analyzed. It is hoped that these results will encourage the use of citation searching as an adjunct to MEDLINE searching. 


\section{METHODS}

\section{Search Data}

One hundred and two mediated searches were collected from four health sciences libraries, each of which serves a teaching hospital. Due to 13 nonreturns, only 89 topics were usable for the study. Two-thirds of the searches were collected from the Alfred Taubman Medical Library at The University of Michigan. All searches were user-initiated. The usual fees were charged for the MEDLINE search. These were real searches motivated by actual information needs. In all cases, when a mediated search was requested on MEDLINE, the user was asked if he or she would be interested in receiving additional items on the same topic from a citation search and if the individual was willing to evaluate the search results for the use of the study. When their cooperation was obtained, a free citation search for each query was supplied. Patrons were asked to supply bibliographic information on one or two "seed" papers which were relevant to the topics to be searched. For each topic, two parallel searches were done by librarians, one on MEDLINE and the other SCISEARCH, the online version of Science Citation Index, which provides citation searching capability on biomedical literature. The MEDLINE printout together with the printout derived from the free citation search was presented to the patron for evaluation.

Following the normal search routines the librarian used either user-suggested terms or other appropriate terms and medical subject headings to access MEDLINE. A citation search produces papers citing a "seed" document supplied by the user. Therefore successful retrieval depends on at least one good "seed" paper. Although there is no objective method to determine its quality in terms of retrieval, an obvious characteristic is its age. A recently published paper would have little chance of being cited. In this study, the average age of the "seed" papers was 10.7 years with a median and mode of 9 and 5 years, respectively. Sixty-two percent of the "seed" documents supplied were published within the previous 10 years, and $52 \%$ were between 4 to 12 years. Thus there was an adequate time lapse for citation to occur.

An important technical point is that for successful citation retrieval, the proper form of the "seed" paper should be checked online using a systems feature "EXPAND." Unfortunately, this procedure was not followed consistently by every searcher. In at least 8 cases, the citation format was not verified rendering no citation retrieval in some searches. Moreover, although the Social SCISEARCH could have been used for several topics, this study was limited to results from SCISEARCH only. Finally, the librarians participating in this project have had many years of searching experience, however, there was no way to control the variations which are known to be introduced by the use of different searchers.

The 89 search requests submitted to health science libraries reflected a wide range of biomedical and other health related topics. Requests came mainly from the clinical faculty, house officers, biomedical researchers, and nurses. Most 
of the queries related to clinical practice and biomedical sciences. There were also queries on other areas such as behavioral problems associated with pregnancy, and child development.

For equitable comparison, data analysis was limited to only those records indexed by both databases. Due to the way each database is structured for content retrieval, a search on a given topic may retrieve a number of items from one file, some of which may not be retrievable from the other database. Yet, if these items resided in both databases, they were included in the assessment of their relative retrieval performance. A common pool of items was established first by the elimination of all pre-1974 items, since the citation database started publication in 1974. Then a list of common journals was identified. Only those items published in these journals were considered for the study. Finally, MEDLINE selectively indexes a group of journals which are indexed by the citation index, in addition to the exhaustive indexing of a large number of core journals. A random check of $10 \%$ of the retrieved papers unique to the citation database showed that less than $3 \%$ of these were not indexed in MEDLINE. As shown in the following section, citation searching retrieved only $1 / 4$ of the total 5558 items unique to each of the databases. Thus, the maximum number of items retrieved which might not have been contained in both databases is quite small. Hence, no attempt was made to identify and eliminate these papers.

\section{Output Evaluation}

Two printouts from each search pair were evaluated by the patron who originated the search to ensure the judgment made in actual information encounters was realistic. The patrons evaluated each printed citation by assigning one of three scores:
A for "relevant" (R) to the query,
B for "partially relevant" (pR) to the query,
C for "not relevant" (NR) to the query.

In the analysis, assignments of $\mathrm{R}$ and $\mathrm{pR}$ were collapsed in a single "all relevant" group as opposed to the "not relevant" group. Relative recall ratios were computed based on the usual "union of output," namely, the number of relevant items retrieved from either database versus all unique relevant or partially relevant items retrieved from both searches. Precision is computed as the ratio of the number of relevant items retrieved as compared with the total number of items retrieved.

\section{RESUlts}

\section{Overall Performance by Term and by Citation Searching}

Slightly less than $5 \%$ of the 5836 unique items, whether relevant or not, were retrieved by both search methods. Overall, MEDLINE achieved an average recall of $77 \%$ and precision of $56 \%$. Citation searching produced $33 \%$ average 


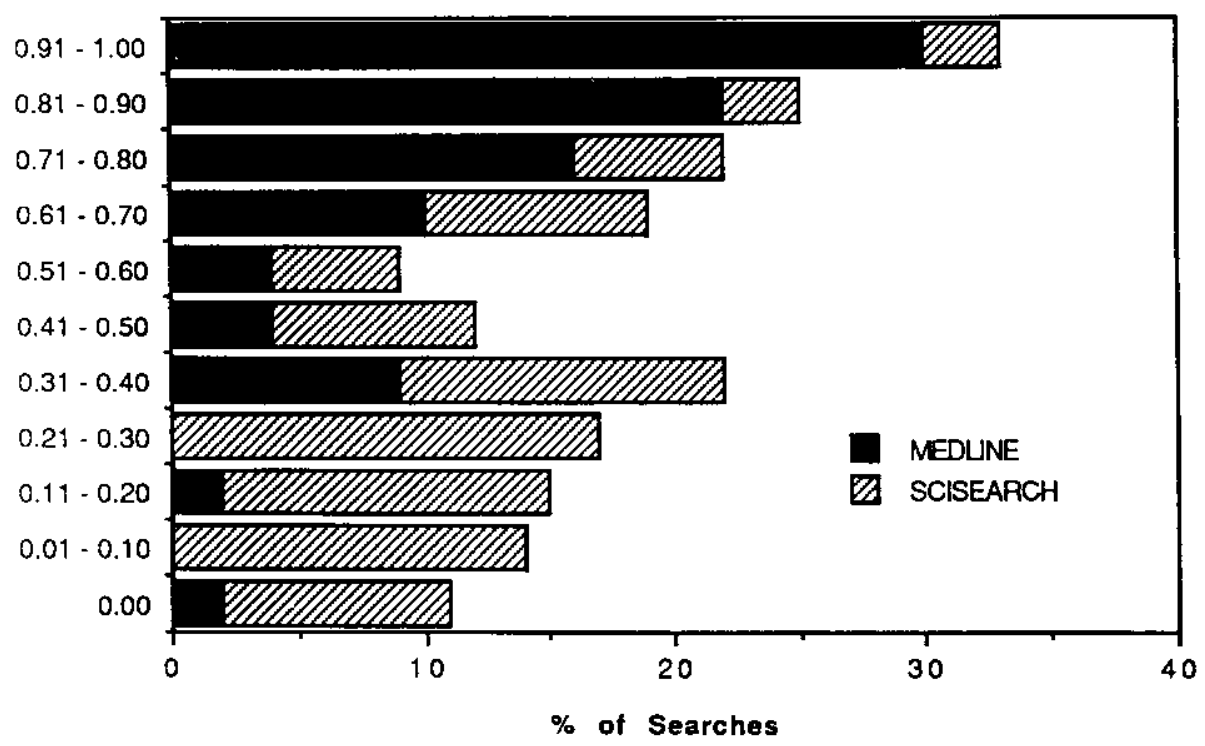

FIG. 1. Distribution of relevant retrieval from MEDLINE and SCISEARCH.

recall with $58 \%$ precision. Figure 1 shows that, as expected, the majority of biomedical literature could be retrieved from MEDLINE by search terms. If searching is limited to MEDLINE, $68 \%$ of all searches achieved $70 \%$ or higher recall, while $66 \%$ of the citation searches fall below a $40 \%$ relative recall. If only the definitely relevant items were tallied, $84 \%$ of the MEDLINE searches achieved above $60 \%$ recall, and $68 \%$ of those from citation were below $40 \%$ recall. The median recall and precision were 27 and $70 \%$, respectively.

Even though recall and precision are standard performance measures of retrieval effectiveness, their interpretation must take into consideration that they are highly skewed distributions. The mean is often greatly inflated by one or two searches with high values. The median should also be taken into consideration for a more accurate representation. Another concern is that these two measures are based on results of individual searches. Although most requestors were not interested in searching exhaustively on their topics, still a wide range of output size was found. For example, two searches retrieved 2 records each, while 472 unique items were retrieved for another topic. Therefore, the sensitivity of a measure based on search-wise level of analysis is limited. Others have suggested that retrieval analysis be based on the relevance of each item retrieved by a search $(I I)$. The item-wise analysis seeks to explain the impact of a given variable on the odds that retrieved items are relevant as opposed to not relevant.

To do this, the log cross ratio analysis was used to determine the relevance odds of items retrieved only from the MEDLINE file as opposed to using citation searching (12). Since only $4.8 \%$ the items were retrieved by both search 
modes, these are analyzed separately in the next section. Two variables were identified. Each must contain only two values. As a result, a $2 \times 2$ table was set up. The independent variable was the search method, with term searching and citation searching as the only two values. The dependent variable was the relevance judgment made by users on each item retrieved. The "all relevant" $(\mathrm{R}+\mathrm{pR})$ value was juxtaposed with the "not relevant" (NR) category. Each item must fit into only one of the four resulting categories.

An answer to the following question was sought: what are the odds that retrieved items be relevant or partially relevant as opposed to not relevant if they are retrieved only from term searching and not found in citation searching as opposed to only from citation searching? There were 5558 items retrieved by one of the two search modes only. MEDLINE retrieved 4092 of them, 2277 of which were judged relevant or partially relevant, while 1815 were judged not relevant. Similarly, of the 1466 retrieved by citation searching alone, 643 were judged relevant or partially relevant and 823 not relevant. The relevance odds were computed as follows:

Searching MEDLINE: $2,277 / 1,815=1.255$

Searching SCISEARCH: $643 / 823=0.78$

Odds Ratio: $1.254 / 0.78=1.609$

Ln Ratio: 0.476

Standard Error: 0.061

$t$ value: 7.77

Since the $t$ value exceeds 2 , this is a statistically significant result at $95 \%$ significance. It indicates that items retrieved from MEDLINE representing searching by terms are $61 \%$ more likely to be relevant or partially relevant as opposed to not relevant. In other words, if one had to choose one of the two search methods, the odds for a retrieved item to be judged at least partially relevant are 8 to 5 in favor of term searching from MEDLINE. Overall descriptor-based retrieval out-performs citation-based retrieval by a factor of 1.61 if items retrieved by both methods were excluded from consideration.

\section{Unique Contribution by Citation Searching}

A more cogent question here is to what extent can citation searching contribute to a MEDLINE search? Four percent of the topics searched produced no retrieval from citation retrieval at all, and $10 \%$ derived no additional relevant items from citation searching. Thus $85 \%$ of the topics searched added at least one relevant item otherwise missed if searching was limited to using MEDLINE alone.

Among 1466 of the 5836 unique papers added to the total pool retrieved by the 89 topics, 326 items were judged relevant, 317 judged partially relevant. Overall $44 \%$ of the added items from citation relationships were judged to be at least partially relevant, and $22 \%$ were definitely relevant to the topics sought.

Focusing on the individual searches, an average of $24 \%$ was gained in recall 
TABLE 1

Distribution OF RECALL DERIVED FROM ADDITIONAL Citation Retrieval

\begin{tabular}{cc}
\hline $\begin{array}{c}\text { Recall } \\
(\%)\end{array}$ & $\begin{array}{c}\text { Searches } \\
(\%)\end{array}$ \\
\hline $0-0$ & 10 \\
$01-10$ & 20 \\
$11-20$ & 25 \\
$21-30$ & 14 \\
$31-40$ & 11 \\
$41-50$ & 5 \\
$51-60$ & 8 \\
$61-70$ & 3 \\
$71-80$ & 0 \\
$81-90$ & 2 \\
$91-100$ & 2 \\
\hline
\end{tabular}

Note. All relevant recall: mean $24 \%$, median $19 \%$; precision: mean $56 \%$, median $63 \%$. Defi-

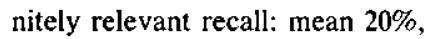
median 12\%; precision: mean $30 \%$, median $34 \%$.

per query from citation retrieval, and among the added items, $56 \%$ were judged to be at least partially relevant. Table 1 showed that the distribution is highly skewed. Ignoring the first line of those topics with no citation retrieval, in $20 \%$ of the topics, citation retrieval added $1-10 \%$ relevant items to the search as a whole; $59 \%$ of the searches gained up to $30 \%$ recall. Thus, while many relevant papers could be retrieved by the linguistic links between terms and the document content they represent, citation retrieval taps a small group of relevant sources not easily identified by semantic relationships.

The next question is what is the tradeoff in terms of effort in scanning another set of retrieval? The distribution of additional items per search ranges from 1 to 240 . Since the mean is not a sensitive representation for the skewed distribution, a further breakdown is shown in Table 2. A high proportion of the search topics had only a few items added. Data in Table 2 are read as follows: for $19 \%$ of the topics, 1 to 2 items were added by citation searching. These items contributed $4 \%$ to the total search and $46 \%$ of them were judged to be partially relevant or relevant. Cumulating the first three lines, for $42 \%$ of the topics, less than 7 added items were retrieved, and approximately half were relevant to the topic sought. These would have been missed if the citation database were not consulted.

The proportional distribution of recall and precision is inconsistent. Most of the recall values are low, indicating that the additional search added only a few 
TABLE 2

Distribution of InCREMENTAL Citation Retrieval vs Additional Recall and Precision

\begin{tabular}{cccc}
\hline $\begin{array}{c}\text { No. of added } \\
\text { documents }\end{array}$ & $\begin{array}{c}\text { Searches } \\
(\%)\end{array}$ & $\begin{array}{c}\text { Mean } \\
\text { recall }\end{array}$ & $\begin{array}{c}\text { Mean } \\
\text { precision }\end{array}$ \\
\hline $1-2$ & 19 & 0.04 & 0.46 \\
$3-4$ & 11 & 0.26 & 0.78 \\
$5-6$ & 12 & 0.19 & 0.58 \\
$7-8$ & 2 & 0.60 & 0.86 \\
$9-10$ & 5 & 0.15 & 0.59 \\
$11-12$ & 7 & 0.23 & 0.59 \\
$13-14$ & 7 & 0.16 & 0.63 \\
$15-16$ & 6 & 0.20 & 0.37 \\
$17-18$ & 4 & 0.21 & 0.36 \\
$19-20$ & 2 & 0.17 & 1.00 \\
$21-22$ & 5 & 0.37 & 0.67 \\
$23-24$ & 0 & 0.00 & 0.00 \\
$25-26$ & 1 & 0.44 & 0.58 \\
$27-28$ & 0 & 0.00 & 0.00 \\
$29-30$ & 2 & 0.41 & 0.83 \\
$31-40$ & 5 & 0.26 & 0.36 \\
$41-50$ & 1 & 0.30 & 0.50 \\
$51-60$ & 1 & 0.20 & 0.57 \\
$61-240$ & 10 & 0.66 & 0.38 \\
\hline
\end{tabular}

relevant items. On the other hand, except for the searches with 15 or more added items, the precision ratio is above the $50 \%$ mark, indicating that over half of these items were judged at least partially relevant. In sum, the added effort and expense of searching a citation database produces a second set of references to be scanned, which in most cases is small and half of the items in the set are likely to be relevant. This is particularly noteworthy since the search requests collected in medical libraries are generally aimed to achieve high precision.

\section{Overlap Retrieval}

An overlap or intersection set contains items retrieved by both term searching and by citation searching. From a total of 5836 unique items retrieved, only 278 (5\%) overlap retrievals were found. Twenty-eight percent of all the searches produced no relevant nor non-relevant overlap item. Of the remaining $72 \%$, over two-thirds had less than 7 overlap items, and $42 \%$ had only $1-2$ common items. Although there were 1 to 60 overlap items in each set, an average of 6 common items per query was found. Similar findings have been noted in other studies.

Examining the extent of relevant overlap shows that of the 278 overlap items, 190 (or 68\%) were judged "relevant" (R) and 240 (or 86\%) were at least 


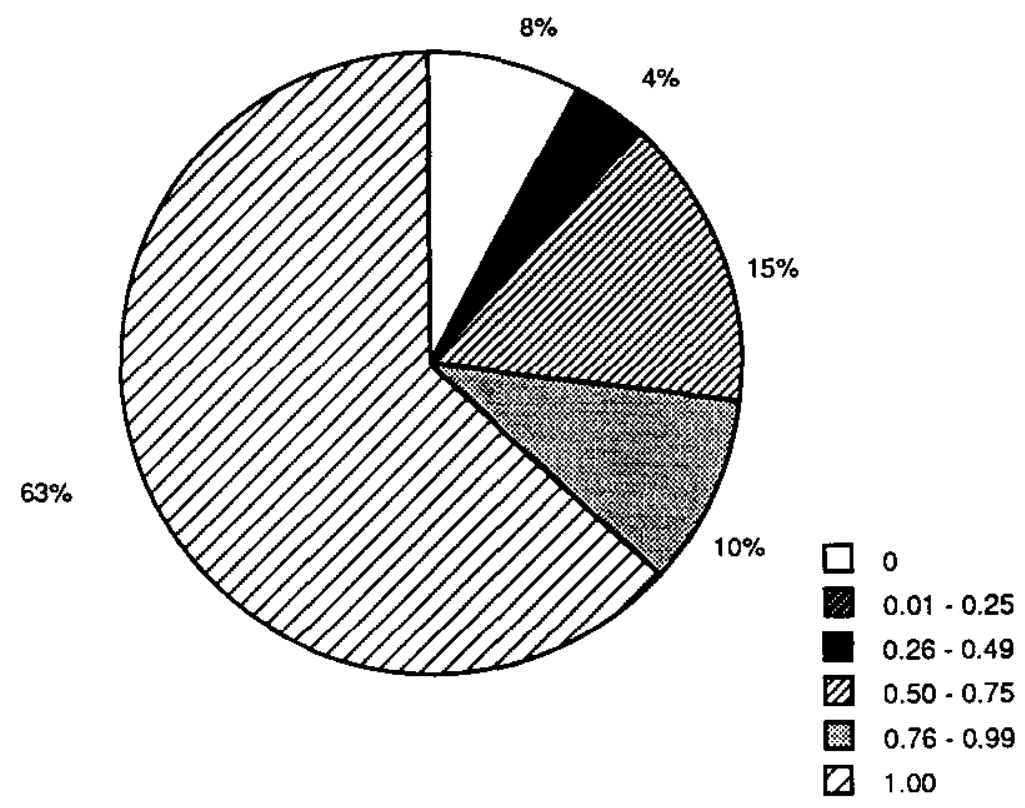

FIG. 2. Distribution of precision values of overlap sets.

"partially relevant" $(\mathrm{R}+\mathrm{pR})$. Figure 2 shows that of the topics with one or more overlap items, $92 \%$ had at least one relevant or partially relevant item; all overlap items in $63 \%$ of the topics searched were partially relevant or relevant and at least half of the overlap items in $88 \%$ of topics were relevant. With the exception of one topic, all overlap sets contain at least one definitely relevant item. The duplicate retrievals from conducting a search in two databases comprised only a scant $13 \%$ of relevant materials, but it is important to note that an average of $81 \%$ of the overlap set were relevant. Table 3 shows that 2-4 rows sums to over $3 / 4(78 \%)$ of the search topics. They had less than 6 (relevant or not relevant) overlaps and $67 \%$ of them had 1-4 relevant items. There was an exceptional query with 60 overlap items, of which 55 were at least partially relevant and 41 of them definitely relevant. Therefore, although few common items were found by terms and by citations, most of the overlaps were judged to be relevant.

What were the odds of an item retrieved for the same question to be judged relevant or partially relevant if it were retrieved from (1) using term searching in MEDLINE, (2) using citation searching in SCISEARCH, or (3) using both methods and retrieved in both cases? The relevance odds were computed in two ways. Two concepts introduced by Saracevic and Kantor were used, namely, "normal relevance" and "strong relevance" (7). For "normal relevance," items judged relevant and partially relevant were pooled to compare with those judged not relevant $(\mathrm{R}+\mathrm{pR}) /(\mathrm{NR})$. For "strong relevance," only relevant items, 
TABLE 3

Distribution of the Relevant Overlap Retrieval

\begin{tabular}{cccc}
\hline $\begin{array}{c}\text { No. of } \\
\text { documents }\end{array}$ & $\begin{array}{c}\text { Total } \\
\text { retrieval } \\
\text { (\% searches) }\end{array}$ & $\begin{array}{c}\text { All } \\
\text { relevant } \\
\text { (\% searches) }\end{array}$ & $\begin{array}{c}\text { Definitely } \\
\text { relevant } \\
\text { (\% searches) }\end{array}$ \\
\hline 0 & 42 & 38 & 10 \\
$1-2$ & 25 & 25 & 48 \\
$3-4$ & 11 & 10 & 17 \\
$5-6$ & 2 & 4 & 8 \\
$7-8$ & 6 & 0 & 2 \\
$9-10$ & 4 & 6 & 4 \\
$11-12$ & 0 & 2 & 2 \\
$13-14$ & 4 & 2 & 2 \\
$15-16$ & 4 & 2 & 2 \\
$17-18$ & 2 & 2 & 4 \\
$19-60$ & 6 & 5 & 2 \\
\hline
\end{tabular}

those preferred by requestors, were compared with not relevant items, R/NR. The latter imposed a more rigid requirement to the relevance odds.

The results are shown in Table 4. The relevance odds of retrieving an item judged to be relevant or partially relevant as opposed to not relevant using only MEDLINE increased by a factor of 1.36 . The previous computation of relevance odds in Section 1 included only those records retrievable from MEDLINE and not retrievable from the citation index. Data in Table 4 were computed as if only MEDLINE were used even if some records could be retrieved by citation searching. If only SCISEARCH were used, the odds were about even (1.03). These were contrasted with the odds that an item retrieved by both methods be at least partially relevant as opposed to not relevant were 6 to 1 , a dramatic jump from either one of the two databases. In other words, it is 6 times more likely that an item appearing in both printouts be relevant or partially relevant.

TABLE 4

Relevance OdDS for THE RETRIEVAl FROM MEDLINE ONLY, FROM SCISEARCH ONLY, AND FROM OVERLAP

\begin{tabular}{lccc}
\hline & $\begin{array}{c}\text { MEDLINE } \\
\text { only }\end{array}$ & $\begin{array}{c}\text { SCISEARCH } \\
\text { only }\end{array}$ & $\begin{array}{c}\text { Overlap } \\
\text { from both }\end{array}$ \\
\hline Normal relevance & 1.36 & 1.03 & 6.32 \\
Strong relevance & 0.81 & 0.61 & 5.18 \\
\hline
\end{tabular}

Note. Normal relevance computed as $(\mathrm{R}+\mathrm{pR}) / \mathrm{NR}$. Strong relevance computed as R/NR. 
Similarly, if "strong relevance" is considered, the odds that items retrieved be definitely relevant are 5 times greater if it were an overlap item. This is convincing evidence that although there is only about $10 \%$ overlap from accessing both databases in the biomedical literature, the chances for relevance increase overwhelmingly for those items found in both outputs. The odds for overlap items to be definitely relevant as opposed to not relevant were equally high. In other words, an overlap item is 6 times more likely to be at least partially relevant and 5 times more likely to be definitely relevant than one retrieved from only one database. Practical use of this information is clear. If only a few items are needed, a short cut method would be to identify items retrieved from both MEDLINE and SCISEARCH.

\section{Characteristics of Search Topics}

In an attempt to gain some insight into the types of queries which could benefit most from the option of citation searching, the content of the queries was examined in light of three arbitrarily determined levels of recall and precision. Each search topic was classified into one of the 9 cells and the queries were distributed among 9 categories. For example, one cell contains queries which achieved high recall and high precision. Each group of topics was examined for any consistent characteristics. Twenty-six percent of the queries belonged to the largest group with relatively high recall and precision. Although there were a variety of topics, they appeared mostly of well-defined scope. For example, the search topic "ectopic thyroid tissues within the left ventricular heart" produced 23 citing papers all of which were judged relevant. Similarly, an interdisciplinary subject on the "nursing care of the elderly" retrieved all 6 relevant papers while MEDLINE produced 22 papers, 3 of which were relevant. As far as one is able to discern, citation searching was least effective in queries that were very specific and tended to lie within a narrow scope. This cursory examination corroborates with earlier studies that queries with interdisciplinary slant appeared to derive the most benefit from citation searching (13). An important caveat on this qualitative analysis is that there are several confounding factors including the inconsistent verification of the bibliographic format of the "seed" papers by all searchers and the quality of the "seeds" supplied. Except for interdisciplinary areas, this study is inconclusive on the types of search topics which could benefit most from a citation search.

\section{Discussions}

Two methods of topical searching are available. One is the use of appropriate terms and the other by relevant citation linkages. The underlying assumption is that they are based on two different criteria of retrieval which in turn represent two distinct types of subject relevance. They complement each other in retrieving relevant materials. This study showed that citation searching is a useful adjunct to MEDLINE in increasing the number of relevant items retrieved, as well as in identifying a few items of definite relevance. While MEDLINE has 
been and will continue to be the most used discipline-based database for the literature of the health sciences, citation retrieval appears to tap another type of relevance not dependent on the semantic relation existing between concepts and terms used to represent them. Thus it could access a pool of papers well beyond the topic sought. Given a paper known to be relevant to a search topic, the mechanics of the search are relatively straight forward. It requires no subjective interpretation on the part of the searcher. An average search can be expected to add $24 \%$ relevant material with a median of $19 \%$ by including citation searching as part of the strategy. This finding is particularly significant in view of the fact that the majority of the participating patrons requested only a few useful items on the topic searched. It should be emphasized that fewer than 7 new items were added to $42 \%$ of the search topics, and about half were relevant. Thus in the health sciences arena, an added citation search can have a definite payoff.

The most astonishing finding from this study is that the odds that overlap items are partially relevant or relevant as opposed to not relevant are 6 times greater than items retrieved using only one of the search methods. The relevance odds from retrieving an item of definite relevance as opposed to one that is not relevant using both methods rather than one were 5 times greater. In the past, while broad agreement exists that overlap items from multiple retrievals tend to be relevant this study provides strong confirming data to corroborate these earlier indications. Moreover, the extreme low overlap further suggests that there is a fundamental difference in the criteria inherent in these two approaches to topical retrieval.

What are the practical implications of the use of citation searching for the health professional? While MEDLINE is well known, SCISEARCH is definitely an infrequently used information resource. In the past, one major barrier has been its high cost. The hourly rate is $\$ 159.00$ per connect hour. For those libraries with the hardcopy of the Science Citation Index the rate is reduced to $\$ 63.00$ per hour. Even at the lower rate, one dollar per minute is considerably higher than most MEDLINE searches would cost. However, it is now more accessible in its CD-ROM format. There is no up-front cost for the use of CDROM for the searcher even though one may need to search several disks for longer coverage. Compared with the modestly priced individual MEDLINE accounts which allow many physicians to access MEDLINE at their convenience at home or at their offices, the CD-ROM product is usually a resource subscribed to by the library. For the clinician in practice, citation searching would mean a trip to a medical library or an order for a mediated search by the librarian. Thus the cost in terms of effort would also pose as a barrier to use.

As far as the fear that too much effort would be wasted on an additional search, a quick-and-dirty strategy is to check the number of citations retrieved displaying or printing only those with relatively fewer citing items. Although the underlying principle is unclear, half of the items associated with fewer citations were found to be at least partially relevant. 
A recent paper reported a spot check of the time lag between the publication of journal articles and their availability online between MEDLINE and SCISEARCH (14). The online version of the citation index was at least one issue ahead for 19 out of 30 journal titles compared. Since the citation index is generated without the time-consuming task of human indexing, it has the distinct advantage of timeliness of access as long as searching is done online. For example, citations appeared in SCISEARCH were 3 issues earlier than MEDLINE for Lancet and 2 issues ahead for both JAMA and NEJM.

However, citation searching is not without its faults. Relevant retrieval relies solely on good "seed" documents. A very recent "seed" paper is handicapped since it does not yet have the opportunity to be cited by as many articles as an older one, if at all. Even if it were cited, the relevant portion of the cited paper is unspecified. In this study, although most of the "seed" documents had ample opportunity to be cited, "seed" papers which were published more than 12 years tended to produce fewer relevant retrievals. Patrons may not always give enough information for the searcher to identify the article. In one case, a paper published in 1941 was given. Even though the searcher failed to verify the citation, the research team could not find any paper by that author which could possibly be the intended "seed" paper. Moreover, their bibliographic format must also be represented accurately in the search string. For example, no hits will result from a search statement such as " $\mathrm{CR}=$ COLLINS FS 1989, V44, P1, AM J HUM, GENET," whereas at least 19 citing references will be retrieved by "CR = COLLINS FS, 1989, V44, P1, AM J HUM, GENET." A recent check with a test version of the CD-ROM product of the Science Citation Index showed that the new software is relatively easy to use and is more forgiving than the online version. Unfortunately, the search features of SCISEARCH are still not as well known to some health science librarians. Although the experiment mimics realistic mediated searching conditions, the citation retrieval results reported here is likely to be suboptimal. In sum, successful citation searching does rely on good "seeds" and proper searching procedures.

Finally, although the usefulness of the overlap items is obvious, currently there is no easy way to identify them other than scanning the two printouts. As of this writing, DIALOG has introduced the duplicate records removal feature on many databases. Sorting the merged list allows one to spot the duplicates. Unfortunately for the biomedical literature user, this convenience is limited only to the use of MEDLINE via DIALOG. Design of future interface softwares should incorporate the ease of duplicate identification.

\section{ACKNOWLEDGMENTS}

The author gratefully acknowledges the help from the staff of the Alfred Taubman Medical Library of the University of Michigan, the Medical Library of the Cleveland Clinic Foundation, the Medical Library of the McLaren Hospital, and the Medical Library of Rush University. Without their cooperation, this study would not have been possible. 


\section{REFERENCES}

1. Haynes, R. B., McKibbon, K. A. Fitzgerald, D., Guyatt, G., Walker, C. J., and SACKETT, D. L. How to keep up with the medical literature. V. Access by personal computer to the medical literature. Ann. Intern. Med. 105, 810 (1986).

2. McKibbon, K. A., Haynes, R. B., Dilks, C. J. W., Ramsden, M. F., Ryan, N. C., Baker, L., Flemming, T., and Fitzgerald, D. How good are clinical MEdLiNE searches? A comparative study of clinical end-user and librarian searches. Comput. Biomed. Res. 23, 583 (1990).

3. Dickersin, K., Hewitt, P., Mutch, L., Chalmers, I., and Chalmers, T. C. Perusing the literature: Comparison of MEDLINE searching with a perinatal trials database. Controlled Clin. Trials 6, 306 (1985).

4. Haynes, R. B., McKibbon, K. A., Walker, C. J., Ryan, N. C., Fitzgerald, D., and RAMSDEN, M. F. Online access to MEDLINE in clinical settings: A study of use and usefulness. Ann. Intern. Med. 112, 78 (1990).

5. PAO, M. L., AND WORTHEN, D. B. Retrieval effectiveness by semantic and pragmatic relevance. J. Am. Soc. Inf. Sci. 40, 226 (1989).

6. PAo, M. L., AND Fu, T. W. Title retrieved from MEDLINE and from citation relations. Proc. Am. Soc. Inf. Sci. 22, 120 (1985).

7. Saracevic, T., and Kantor, P. A study of information seeking and retrieving: III. Searchers, searches, and overlaps. J. Am. Soc. Inf. Sci. 39, 197 (1988).

8. MCCAIN, K. W. Descriptor and citation retrieval in the medical behavioral sciences literature: Retrieval overlaps and novelty distribution. J. Am. Soc. Inf. Sci. 40, 110 (1989).

9. Katzer, J., McGill, M. J., Tessier, J. A., Frakes, W., and DasGupta, P. A study of the overlap among document representation. Inf. Technol. Res. Dev. 2, 261 (1982).

10. Salton, G. Automatic indexing using bibliographic citations. J Doc. 27, 98 (1971).

11. Saracevic, T., Kantor, P., Chamis, A. Y., and Trivison, D. A Study of information seeking and retrieving: I. Background, and methodology. J. Am. Soc. Inf. Sci. 39, 161 (1988).

12. Fleiss, J. L. "Statistical Methods for Rates and Proportions," 2nd ed. Wiley, New York, 1981.

13. Goffman, W., And Pao, M. L. Retrieval of biomedical information for emerging interdisciplinary problems. In "Proceedings of the 4th International Congress on Medical Librarianship," pp. 39-50, 1980.

14. SNOw, B. SCISEARCH changes: Abstracts and added indexing. Online 15(5), 102 (1991). 